\title{
A Neurobiologically Inspired Model of Working Memory Based on Neuronal Synchrony and Rythmicity
}

\author{
Jacques Sougné \& Robert M. French \\ Department of Psychology \\ University of Liège, 4000 Liège, Belgium \\ $\{$ J.Sougne, rfrench $\} @$ ulg.ac.be
}

\begin{abstract}
The connectionist model of reasoning presented here, INFERNET, implements a working memory that is the activated part of long-term memory. This is achieved by making use of temporal properties of the node spikes. A particular solution of the problem of multiple instantiation is proposed. This model makes predictions that have been tested experimentally and the results of these experiments are reported here. These results would seem to challenge modular models of memory.
\end{abstract}

\section{$1 \quad$ Introduction}

Connectionist models of working memory face two main problems. The first is the binding problem; the second is the problem of multiple instantiation. The model presented here draws its inspiration from neurobiology in an attempt to solve these problems. Different aspects of the same stimulus are not processed by the same neurons. The brain has to link together these various aspects (e.g., color, contours, movement) in order to differenciate them from other objects. This is referred to as variable binding and the present model achieves it through the use of temporal synchrony. In short, when one node (i.e., a group of neurons) fires in synchrony with another, they are temporarily bound together. This technique can be used to successfully represent n-ary predicates. This idea has been applied to "reflexive" reasoning [15], to natural language parsing [7], to analogical inferences [8], and to deductive reasoning [18]. Multiple instantiation involves simultaneous use of the same parts of the knowledge base in different ways. Connectionist models that define working memory as the activation of parts of representations in long-term memory must explain how multiply-instantiated entities are handled. In this paper we describe one such model, INFERNET, show how it simulates various aspects of human working memory, and demonstrate how it represents multiply instantiatiated concepts. Predictions will follow and experimental data will be presented which confirm predictions made by the model. 


\section{INFERNET description}

\subsection{Concepts and attributes}

INFERNET is a connectionist model using integrate-and-fire nodes. Each concept is represented by a cluster of nodes firing in synchrony (figures 1, 2). Concepts are

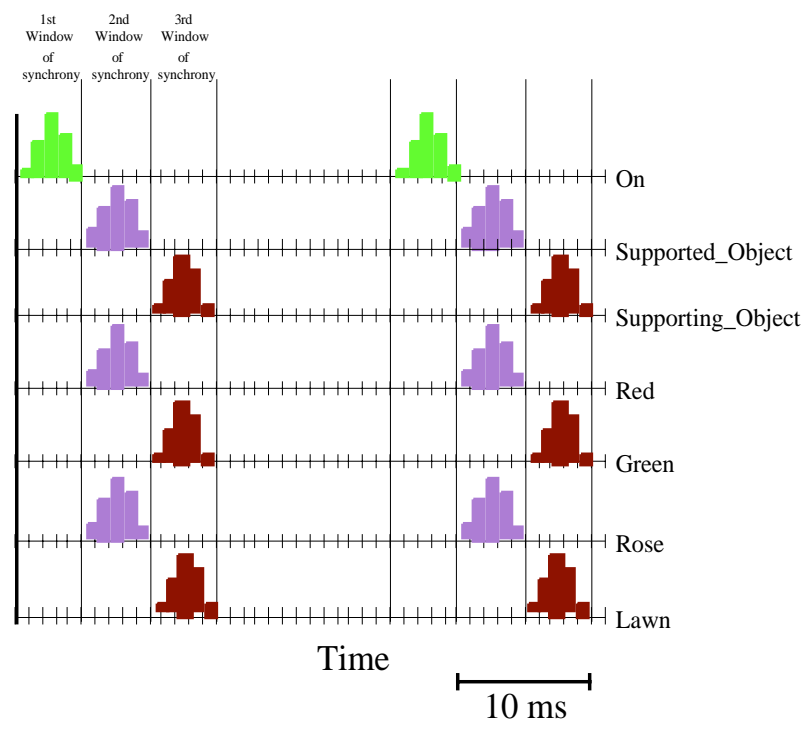

Figure 1: The "red rose on the green lawn" requires binding of concepts "red" and "rose" with the role "Supported_Object", followed by "green" and "lawn" with the role "Supporting_Object.

bound together by synchronous firing. For example, to represent the concept "red rose", nodes belonging to "red" must fire synchronously with nodes belonging to "rose" (figure 1). There is neurobiological evidence for considering synchrony as a possible binding mechanism in the brain. In particular, synchrony has been observed between distant cells in the same cortical area, between cells in different cortical areas and even between cells in different hemispheres. If a number of different objects make up a scene, distinct windows of synchrony are formed, each associated with a particular object. Individual cells can rapidly change partners of synchrony if the stimulus changes. Moreover the absence of synchronization has been observed to impair cognitive abilities. For a complete discussion on synchronization as a neural binding mechanism, see $[16,14]$.

\subsection{Discrimination}

Discrimination is achieved by successive synchronies, for example, to discriminate a red rose on a green lawn. The nodes belonging to "red" "rose" and "Supported_Object" must fire in synchrony and those corresponding to "green" "lawn" and "Supporting_Object" must also fire in synchrony. Further, these two 
sets of nodes must fire in close succession and after the nodes belonging to "On" for

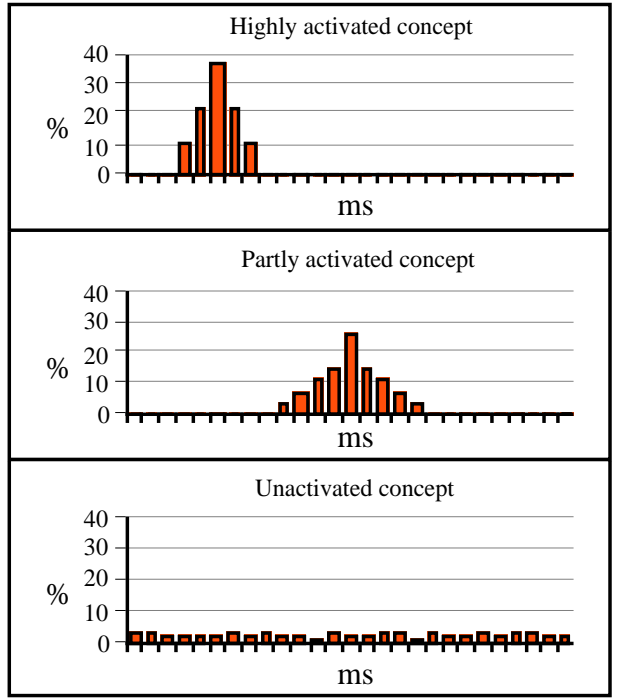

Figure 2: The temporal distribution of node-firing determines if a concept is activated.

"the red rose on the green lawn" to be perceived (figure 1). Engel and al. [6] provide evidence that shows that if several objects are present in a scene, several group of cells are grouped in distinct windows of synchrony.

\subsection{Initial constraints}

A number of neurobiological parameters are involved in this representation that rely on clusters of nodes firing simultaneously. The first is the frequency of oscillation. In INFERNET, as in SHRUTI [15], once a node is activated, it tends (but not necessarily) to fire rhythmically between 30 and $100 \mathrm{~Hz}$. The temporal gap between 2 spikes of a node is therefore from 10 to $33 \mathrm{~ms}$. This corresponds to the observed $30-100 \mathrm{~Hz}$ ( $\gamma$ wave) oscillations of certain types of neurons. These $\gamma$ waves have been observed to be associated with attention [21] and with associative memory [22]. The second key parameter is the precision of the synchrony. According to [17] this precision is between 4 to $6 \mathrm{~ms}$. For [1], the precision is about $5 \mathrm{~ms}$ and depends on the frequency of oscillation.

\subsection{Windows of synchrony as working memory span}

Since concepts are represented as a set of nodes, INFERNET focuses on the distribution of node-firing times. If the firing distribution is tightly concentrated about the mean, the concept is considered to be activated. In figure 2, three distibutions are depicted. The $\mathrm{Y}$-axis represents the percentage of nodes belonging to a concept that are firing, the $\mathrm{X}$-axis shows the time in $\mathrm{ms}$. The top graph shows the distribution of a highly activated concept, all nodes pertaining to the concept fire within $5 \mathrm{~ms}$. The middle graph shows a less activated concepts whose nodes all fire 
within $9 \mathrm{~ms}$. The lower graph shows the distribution of an unactivated concept node. In this last case, too few of the concept nodes fire and what firing there is does not occur within a short interval.

In Figure 1, nodes corresponding to the concept "rose" are firing in synchrony and the firing-time distribution is concentrated around the mean. Nodes pertaining to "rose" fire in synchrony with nodes representing "red". This synchrony is distinguished from the synchrony between nodes pertaining to "green" and "lawn" within the same cycle. Their means are clearly different.

The temporal lag between 2 spikes of a node oscillating at $\gamma$ frequency $(30-100$ $\mathrm{Hz}$ ) is between 10 to $33 \mathrm{~ms}$., and is typically about $25 \mathrm{~ms}$. The precision or width of a window of synchrony is about $5 \mathrm{~ms}$. and is proportional to the frequency [1]. This allows us to approximate the number of windows of synchrony that could be differentiated, i.e. $25 / 5=5$. If we assume that a window of synchrony corresponds to an item or a chunk in working memory, then this puts working memory span at approximately 5 , with a small amount of variance since precision is proportional to oscillation frequency. This corresponds to current estimates of human working memory span. It has been suggested [4], that the traditionally accepted size of working memory (i.e., $7 \pm 2$ items [12]) may be too high. An item can be a word, an idea, an object in a scene or a chunk, i.e., a grouping of items. Similar explanations for the brain's ability to store approximately 7 short-term memory items can be found in $[9,10,15]$.

\subsection{Persistance in working memory}

How can representations be maintained in working memory? The problem with $\gamma$ waves is that they persist only a few hundred milliseconds. This is not long enough to reflect the time taken by people to draw inferences, nor does it correspond to standard estimates of working memory retention time (10 to 20 seconds). For this reason, following [10], $\gamma$ waves in INFERNET occur in bursts which restart every 146 to $333 \mathrm{~ms}$. This corresponds to $\theta$ waves [3 - $7 \mathrm{~Hz}$ ] whose duration can exceed 10 seconds. The resulting temporal firing pattern for a single node is shown in Figure 3. The node shown fires at $50 \mathrm{~Hz}$ for the seven spikes that constitute a burst. This is followed by a resting period of $60 \mathrm{~ms}$. Thereafter, the burst begins again. The burst interval is about $200 \mathrm{~ms}(5 \mathrm{~Hz})$.

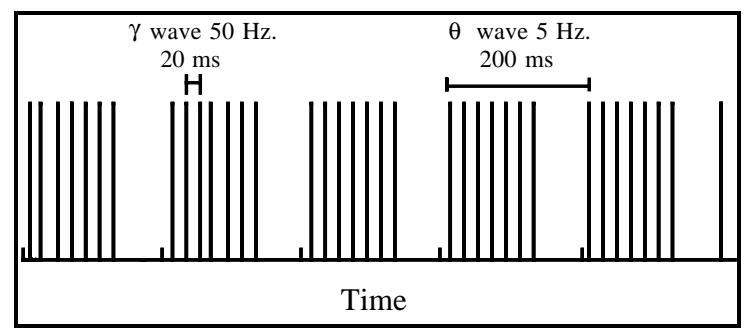

Figure 3: $\gamma$ wave embedded in $\theta$ wave

There is neurobiological evidence for this rhythm in working memory. $\theta$ waves have been observed to be associated with visual short term memory task on a monkey [13]. This wave was maintained as long as attention was required. 


\subsection{Chunking}

Working memory capacity is limited, and chunking increases the amount of information it can contain. In INFERNET, chunking is achieved by two processes:

1. Increasing the number of nodes - and, as a result, the number of concepts firing in synchrony. This is achieved by means of spreading activation.

2. Replacing the content of two or more windows of synchrony by a single one that sums them up. This is achieved by the use of excitatory and inhibitory connections.

\subsection{Interference}

Working memory is affected by interference. For example, when Working memory is successively tested by different words pertaining to the same category, the span decreases during the course of the trial. In INFERNET, concepts pertaining to the same category have nodes in common. Previously memorized items interfere with newly arriving ones if they share common nodes.

\subsection{Memory scan}

Sternberg [20] asked participants to rehearse lists of 1 to 6 items. At a nonpredictable moment, participants received a probe item and were asked to decide as quickly as possible if the probe had been in the list of memorized items. Sternberg found that response time increased linearly according to the number of memorized items. For each additional item, reaction time increased by $38 \mathrm{~ms}$. (see figure 4). Other replications showed a shorter increase of reaction time e.g. [11] found an increase of $22 \mathrm{~ms}$.

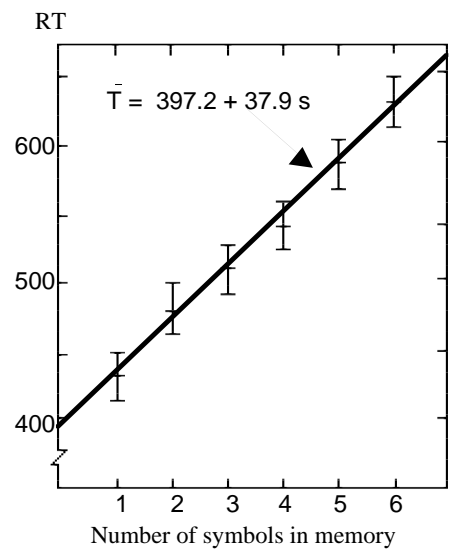

Figure 4 : Memory scan, Sternberg data

A closer look at the Sternberg data shows that, while overall reaction time increases with the number of items memorized, the variance remains constant. It is hard to see how this would be the case if the process was serial. If we consider each 
item as an independent random variable, then the total variance would be equal to the

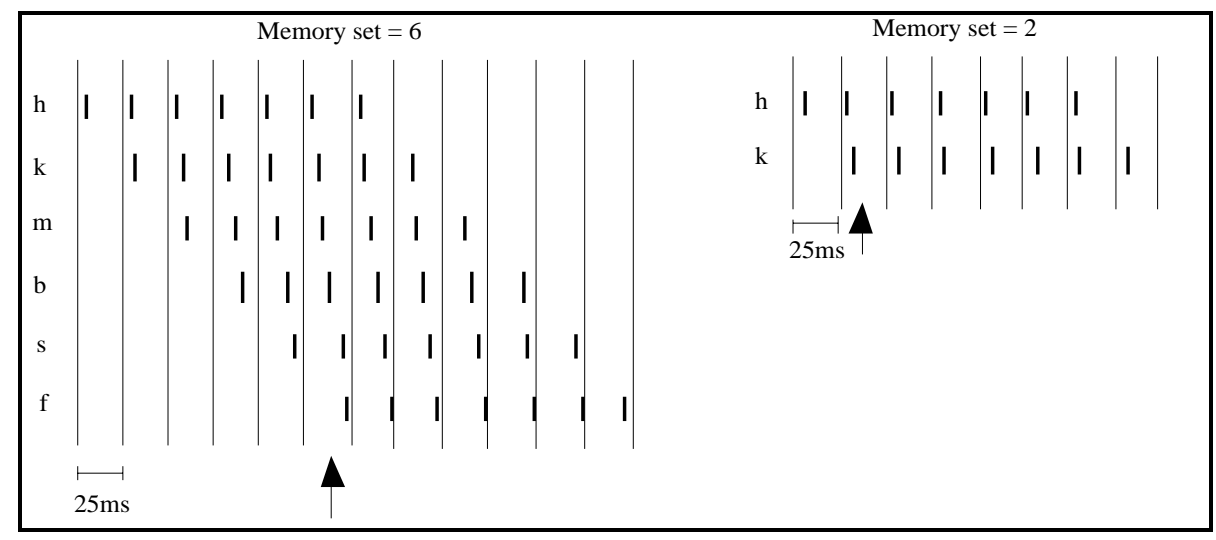

Figure 5 : Simultaneous activation of the memory set and starting point (arrow) of the parallel matching process in INFERNET.

sum of the variances associated with each of the variables. Just as measuring the length of a room by repeated ("serial") measurements with a six-inch ruler will produce a proportionately greater total error than measuring the room with a yardstick or a tape-measure, the same should hold for the Sternberg reaction-time data. If we have a serial process, then not only should the mean reaction time increase, but the variance should as well. But this is not the case.

In contrast to a serial recognition process, which would produce a linear increase in variance as the number of items increases, one solution compatible with INFERNET would produce the uniform variance that we observe in Sternberg's data. The actual implementation of this process is currently in progress. Recognition of previously seen items could involve a multi-phase process:

- Storage: the original items are inserted into memory and are refreshed once every every $\theta$-wave cycle. At this point there is no need for the additional computational resources required by maintaining each item in a window of synchony.

- Simultaneous item activation (figure 5): The probe arrives: "Was the following item in the list?" followed by some item. At this point, the memorized items must be simultaneously activated in working memory. This involves the following serial process: one of the items in the list (for example, "h") will be associated with a particular window of synchrony (for example, the first window) and, thereafter, will appear in that position with every 25-ms cycle of the $\gamma$ wave. Each of the other items in the originally learned list will be activated in the same manner, with its own window of synchrony and once every $25 \mathrm{~ms}$. Once this process is completed - i.e., all of the letters in the list have been assigned to a window of synchrony - only then can the matching process begin. Thus, the length of time required to set up the matching process increases linearly with the number of items in the original list (approximately $25 \mathrm{~ms}$. per item).

- Matching: This process occurs in parallel across all items and therefore takes a constant amount of time, independent of the number of items required in the matching.

As a result, reaction time would be a function only of the setup time required by the system once the probe has been presented. All of the elements must, in fact, be 
retrieved before the matching process can begin. A related explanation can be found in [10], although their work does not involve the problem of uniform variance with an increased number of items to be memorized.

\subsection{Multiple instantiation}

Multiple instantiation involves the simultaneous use of the same parts of the knowledge base in different ways. Knowing that "John is in love with Louise" and that "Louise is in love with John", one can easily infer that they should be happy. To arrive at this conclusion one must instantiate the predicate "is in love with" and the objects "John" and "Louise" twice. Precisely how this is done is the problem of multiple instantiation.

Traditional models (e.g. [2]) that load copies of pieces of knowledge into a working area before transforming them do not have any problem with multiple instantiation. They simply make several copies of the same content from the longterm knowledge base. However, for connectionists models that use the structure of the knowledge base itself as the place where concepts are associated, transformed and where inferences are drawn, multiple instantiation is a serious problem. How can the same part of the knowledge base be associated with different things at the same time without making several copies of the knowledge in question? This question is crucial for connectionist models of working memory. Multiple instantiation poses a significant problem for distributed representations. Two closely related concepts will, in principle, share nodes. If both concepts are needed simultaneously, their common parts must be instantiated twice.

However, some studies [3, 5, 19] show that humans handle at least double instantiation without difficulty. Experimental tasks using single and double instantiation are rare. One example is relational reasoning (three-term-seriesproblem). Singly-instantiated predicates in "A is better than B, C is worse than B" were compared to doubly-instantiated predicates in "A is better than B, B is better than $\mathrm{C}$ " and no difference in reaction times between these two situations was found $[3,19]$. No difference of correctness can be found in [5]. These data seem to demonstrate that performance is not impaired by double instantiation. In other words, this would seem to imply that there is, in fact, no cost in terms of additional processing time for double instantiation. This property will emerge naturally from the underlying assumptions of INFERNET. However, this prediction should be assessed by further experiments.

The present model modifies the frequency of the $\gamma$ wave to enable multiple instantiation. This means that neurons pertaining to a doubly-instantiated concept will oscillate twice as fast as singly-instantiated ones. If we assume a $\gamma$ wave frequency between 30 and $100 \mathrm{~Hz}$., the number of multiple instantiations should be limited to about 3 with little or no additional cost to the system. In this case, singly-instantiated nodes could fire at $30 \mathrm{~Hz}$, doubly-instantiated ones at $60 \mathrm{~Hz}$, and triply-instantiated ones at $90 \mathrm{~Hz}$. Since the maximum oscillatory frequency of the $\gamma$ wave is approximately $100 \mathrm{~Hz}$, anything beyond triple instantiation will require chunking, and this would require extra time. INFERNET therefore suggests that the brain does multiple instantiation by replacing a number of windows of synchrony by a single "chunked" one. If the number of instantiations exceeds 3 , an increase of processing time proportional to the difficulty of chunking should be observed. 


\section{Experiment 1}

When the number of instances increases (beyond 2 or 3 ), a chunking process should reduce the number of instantiations in different windows of synchrony. Sometimes this process is easy, for example, when all instances can be grouped with one proposition. Sometimes it is more difficult, when no single proposition can be found that could chunk all instances. INFERNET predicts that in the latter case reaction time will increase. Experiment 1 will test this prediction.

\subsection{Participants and design}

The 30 participants were undergraduate psychology majors randomly assigned to each of two conditions that differed in their ease of chunking.

\subsection{Material}

Two sets of 4 premises (relational statements), using the same number of words, were constructed.

The easy chunking group set

"Allan is in love with Mary",

"Mary is in love with Allan",

"Peter is in love with Barbara",

"Barbara is in love with Peter".
The hard chunking group set

"Peter is in love with Mary",

"Barbara is in love with Allan",

"Allan is in love with Mary"

"Mary is in love with Peter".

The question asked of participants of both conditions was "Who is happy?" All material was presented by a computer program allowing reaction time to be recorded. Participants' conclusions were recorded manually.

\subsection{Procedure}

Each participant was seated approximately $50 \mathrm{~cm}$ from the monitor. The four premises appeared on the screen simultaneously in a random order. Participants were asked to read these 4 premises and to indicate when they had finished. Then a question appeared on the screen. Reaction time for answering the question was recorded. Before presenting the experimental material, participants received training exercises with the same procedure, but with an arithmetic content.

\subsection{Results}

Table 1 shows reaction times for all correct responses. Differences between groups are significant (Mann-Whitney $\mathrm{Z}=-2.331, \mathrm{p}=0.019$ ).

\begin{tabular}{|l|c|c|}
\hline & Mean & SD \\
\hline Easy chunking group & 3719 & 1025 \\
\hline Hard chunking group & 7778 & 4312 \\
\hline
\end{tabular}

Table 1: Reaction time in ms for hard and easy chunking of multiple instantiation 


\subsection{Discussion}

For the easy chunking group where all relations are reciprocal, subjects can rapidly replace the relation "is in love with" by "love each other", and finally, by "are happy", therefore reducing the number of instantiations. By contrast, in the hard chunking group, subjects need to distinguish items for which the "love each other" relation is true from items for which this relation is false and maintain this distinction in working memory, before enabling the replacement process. This additional process takes time, explaining the significantly higher response times. Dealing with more than 2 or 3 instantiations (in this case, there were 4) seems to require additional processing resources, at least when no single property can be applied to all instances. One might reasonably object, for example, that Peter might not be happy because he was upset that Allan loves the person he loves and that Mary could be upset of the love of Peter. The point is well taken, but in this experiment we only considered response time for participants who replied that both Peter and Mary were happy. In addition, no participants replied that both Peter and Mary were unhappy.

\section{Experiment 2}

For a distributed connectionist model, multiple instantiation will also affect related concepts. Concepts that share properties most likely share something in the neurobiological substrate. The effect of multiple instantiation should be observable when related concepts are used together. The following experiment tests this hypothesis.

One of the key features of distributed connectionist models is that a single concept is represented by a large set of nodes, referred to here as a cell assembly. Moreover, a single node can participate in different cell assemblies. In INFERNET, a concept is represented by a set of nodes firing in synchrony. The distributed nature of each concept implies that closely related concepts have some nodes in common. If two related concepts are needed simultaneously, and if they cannot belong to the same window of synchrony, the nodes that they share must be instantiated twice. In the present experiment the number of closely related concepts was manipulated. The prediction was that if the number of instantiations of shared properties exceeded 2 or 3 , a replacement process would be triggered. This replacement must take time and would be reflected in the subjects' response times.

\subsection{Participants and design}

The 40 participants were undergraduate psychology majors. They were randomly assigned to each of two conditions. These two conditions differed in the number of shared properties of concepts.

\subsection{Material}

Two rules of the type "if...then" (material implication), one for each condition, were constructed. These rules have the same length. The first rule assigned to the group of participants called "distant group" involved rather distant concepts: "If the lumberjack cuts down the oak tree, the farmer's tractor can use the pathway". The 
second rule assigned to the group of participant called "related group" used more closely related concepts: "If the lumberjack cuts down the oak tree, the carpenter can nail the oak boards". In the latter rule, there are 7 concepts related to wood. Four questions for each condition were designed. For the first condition, these were: "The lumberjack cut down the oak tree. What do you conclude?" "The lumberjack didn't cut down the oak tree. What do you conclude?" "The farmer's tractor can use the pathway. What do you conclude?" "The farmer's tractor can't use the pathway. What do you conclude?" For the second condition, they were: "The lumberjack cut down the oak tree. What do you conclude?" "The lumberjack didn't cut down the oak tree. What do you conclude?" "The carpenter can nail the oak boards. What do you conclude?" "The carpenter can't nail the oak boards. What do you conclude?"

The four questions and the rule correspond to the following logical forms: $A \supset B$, $\mathrm{A} ; \mathrm{A} \supset \mathrm{B}, \sim \mathrm{A} ; \mathrm{A} \supset \mathrm{B}, \mathrm{B} ; \mathrm{A} \supset \mathrm{B}, \sim \mathrm{B}$. All material was presented by a computer program allowing response times to be recorded. Participants' conclusions were recorded manually.

\subsection{Procedure}

Each participant was seated approximately $50 \mathrm{~cm}$ in front of the monitor. One of the rules appeared on the screen. Participants were asked to read the rule and to indicate when they had understood it. The rule stayed on the screen during the entire experiment. Questions appeared on the screen, one at the time and in random order. Participants had to answer each question. The computer recorded the time required for them to respond. Before presenting the experimental material, participants received training exercises with the same procedure, but with an arithmetic content.

\subsection{Results}

There were no significant differences in betweeen-group reaction times (Table 2) for each type of inference, Modus Ponens (MP), Denying the antecedent (DA), Affirming the consequent (AC), and Modus Tollens (MT). Only reaction times for equivalent responses were considered.

\begin{tabular}{|c|c|c|c|c|c|c|c|c|}
\hline \multicolumn{2}{c|}{ MP } & \multicolumn{2}{c|}{ DA } & \multicolumn{2}{c|}{ AC } & \multicolumn{2}{c|}{ MT } & \multirow{1}{*}{} \\
\hline & Mean & SD & Mean & SD & Mean & SD & Mean & SD \\
\hline Group related & 4031 & 1135 & 3898 & 982 & 4507 & 1518 & 5062 & 1844 \\
\hline Group distant & 3526 & 1285 & 3685 & 1093 & 4177 & 1829 & 4298 & 1587 \\
\hline
\end{tabular}

Table 2 : Mean reaction time for each inference type

Reaction times for each question presented in succession are considered. Table 3 shows the data (mean and SD) for the first, second, third and fourth question (questions were presented in random order). Only reaction times for equivalent responses were taken into account.

There was a significantly longer reaction time for answering the first question presented in the group "related" (Mann-Whitney $\mathrm{Z}=2.994, \mathrm{p}=0.002$ ). All other differences of reaction time were not significant. Reaction time for reading the rule does not differ significantly among groups for the related group: mean $8058 \mathrm{~ms}$ for the distant group $8243 \mathrm{~ms}$. (Mann-Whitney $\mathrm{Z}=-0.132, \mathrm{p}=0.911$ ). 


\begin{tabular}{|c|c|c|c|c|c|c|c|c|}
\hline \multicolumn{1}{c|}{ 1st Question } & \multicolumn{2}{c|}{ 2nd Question } & \multicolumn{3}{c|}{ 3h Question } & 4th Question & \multicolumn{1}{c|}{ 4hean } \\
\hline & Mean & SD & Mean & SD & Mean & SD & Mean & SD \\
\hline Group related & 4893 & 1426 & 3815 & 807 & 4204 & 1170 & 4322 & 1701 \\
\hline Group distant & 3499 & 1024 & 3737 & 1197 & 4341 & 1960 & 3774 & 1456 \\
\hline
\end{tabular}

Table 3: Mean reaction time by order of presentation.

There is no between-group difference regarding conclusion inferred, as Table 4 shows. Fisher exact probabilities are all not significant $(.5, .7564, .3025, .6693$, respectively).

\begin{tabular}{|c|c|c|c|c|}
\hline & MP & DA & AC & MT \\
\hline Group related & 1.00 & .95 & .85 & .85 \\
\hline Group distant & 0.95 & .95 & .95 & .85 \\
\hline
\end{tabular}

Table 4: proportion of conclusions inferred

\subsection{Discussion}

There are no differences in the time required for participants to read one rule or another, but when they receive the first question, they must encode the rule in a particular way, thereby permitting an inference to be drawn. This encoding requires dealing with multiply-instantiated properties that share the concepts used in the rules. A replacement process is required, "the lumberjack cuts down the oak tree" must be assigned to a unique antecedent object, or window of synchrony. The two different consequents: "The farmer's tractor can use the pathway" and "The carpenter can nail the oak boards" must also be assigned to a single consequent object or window of synchrony. For these consequent parts, there is a crucial difference: the concepts in the sentence "The carpenter can't nail the oak boards" share properties with each other and with those used in the antecedent part of the rule. Multiple instantiations of these shared properties impair the replacement process, thereby increasing the time required to answer the first question. When the following three questions appear, this replacement has already been done, and reaction times no longer differ. The reaction time difference for the first question is not due to the type of question posed. The four different questions appear in random order for each participant and there is no significant difference in between-group reaction time for each of the question types $A$ (MP), A (DA), B (AC) and $\sim \mathrm{B}(\mathrm{MT})$. The only significant difference between the two groups occurs for the first question - when encoding occurs. In addition, the lack of any significant between-group difference related to the conclusions inferred, reinforces the idea that the only difference between groups involves multiple instantiation.

\section{Conclusions}

INFERNET attempts to simulate various aspects of a human working memory defined as the activated part of long-term memory. In particular, INFERNET predicts that multiple instantiation will not require additional processing time as long as the number of instantiations does not exceed 2 or 3 . When the number of instantiations 
does exceed 2 or 3, INFERNET predicts a replacement process which requires additional processing time. The preliminary data reported here would seem to confirm this. INFERNET also predicts that dealing with closely related concepts will require multiple instantiation. The experimental results presented here would seem to confirm this hypothesis. These results seem to support distributed concept representations and challenge modular accounts of memory. For the latter models (e.g. [2]), working memory is distinct from long-term memory and the contents of LTM are loaded into WM when needed. According to modular memory models, multiple instantiation should not increase reaction time. The results reported here contradict this prediction.

\section{Acknowledgments}

This research was supported by the Belgian FNRS Grant D.4516.93 \& PAI p4/19.

\section{References}

1. Abeles, M., Prut, Y., Bergman, H., Vaadia, E. \& Aertsen, A. Integration, Synchronicity and Periodicity. In A. Aertsen (Ed.) Brain Theory: SpatioTemporal Aspects of Brain Function. Amsterdam: Elsevier. 1993

2. Baddeley, A. D. Working Memory. Oxford: Oxford University Press, 1986

3. Clark, H.H. Linguistic process in deductive reasoning. Psychological Review, 1969, 76: 387-404.

4. Crowder, R. G. Principle of learning and memory. Hillsdale: LEA. 1976

5. De Soto, C. B., London, M. \& Handel, S. Social reasoning and spatial paralogic. Journal of Personality an Social Psychology, 1965, 2, 513-521.

6. Engel, A. K., Kreiter, A. K., König, P., \& Singer, W. Synchronisation of oscillatory neuronal responses between striate and extrastriate visual cortical areas of the cat. Proceedings of the National Academy of Science, 1991, 88: 6048-6052.

7. Henderson, J. Description Based Parsing in a Connectionist Network. PhD thesis, University of Pennsylvania, Technical Report MS-CIS-94-46. 1994.

8. Hummel, J. E. \& Holyoak, K. J. LISA: A Computational Model of Analogical Inference and Schema Induction. Proceedings of the Eighteen conference of the Cognitive Science Society. Mahwah,NJ: LEA. 1996.

9. Jensen, O. \& Lisman, J. E. Novel lists of $7 \pm 2$ known items can be reliably stored in an oscillatory short-term memory network: Interaction with long term memory. Learning \& Memory, 1996, 3, 257-263.

10. Lisman, J. E., \& Idiart, M. A. P. Storage of $7 \pm 2$ Short-Term Memories in Oscillatory Subcycles. Science, 1995, 267: 1512-1515.

11. Marsh, G. R. Age differences in evoked potential correlates of a memory scanning process. Experimental Aging Research, 1975, 1, 3-16.

12. Miller, G. A. The Magical Number Seven, Plus or Minus Two. Psychological Review, 1956, 63, 81-97.

13. Nakamura, K., Mikami, A. \& Kubota, K. Oscillatory neuronal activity related to visual short-term memory in monkey temporal pole. Neuroreport, 1992, 3: 117-120.

14. Roelfsema, P. R., Engel, A. K., König, P. \& Singer, W. The role of neuronal synchronization in response selection: A biologically plausible theory of 
structured representations in the visual cortex. Journal of Cognitive Neuroscience, 1996, 8, 603-625.

15. Shastri, L. \& Ajjanagadde, V. From Simple Associations to Systematic Reasoning: A connectionist representation of rules, variables and dynamic bindings using temporal synchrony. Behavioral and Brain Sciences, 1993, 16, 417-494.

16. Singer, W. Synchronization of neuronal responses as a putative binding mechanism. In M. A. Arbib (Ed.) The Handbook of Brain Theory and Neural Networks. Cambridge: MIT Press. 1995.

17. Singer, W. \& Gray, C. M. Visual Feature Integration and the Temporal Correlation Hypothesis. Annual Review of Neuroscience, 1995, 18: 555-586.

18. Sougné, J. A Connectionist Model of Reflective Reasoning Using Temporal Properties of Node Firing. Proceedings of the Eighteen conference of the Cognitive Science Society. Mahwah,NJ: LEA. 1996.

19. Sternberg, R. J. Representation and process in linear syllogistic reasoning. Journal of Experimental Psychology: General, 1980, 109, 119-159.

20. Sternberg, S. High Speed Scanning in Human Memory. Science, 1966, 153, $652-654$

21. Wang, X. \& Rinzel, J. Oscillatory and Bursting Properties of Neurons. In M. A. Arbib (Ed.) The Handbook of Brain Theory and Neural Networks. Cambridge: MIT Press. 1995.

22. Wilson, M. \& Shepherd, G. M. Olfactory Cortex. In M. A. Arbib (Ed.) The Handbook of Brain Theory and Neural Networks. Cambridge: MIT Press. 1995. 